\title{
EXPERIMENTAL VALIDATION OF THE SPECTRAL FIT ALGORITHM USING TISSUE MIMICKING PHANTOMS
}

\author{
T.A. Bigelow, W.D. O’Brien, Jr. \\ Bioacoustics Research Laboratory, Department of Electrical and Computer Engineering,
} University of Illinois, 405 North Mathews, Urbana, Illinois 61801 USA

\begin{abstract}
Characterizing tissue by measuring its physical properties has potential for improving the diagnostic capabilities of medical ultrasound. One parameter that is estimated using the power spectrum of backscattered ultrasound echoes is the characteristic size or correlation length of the tissue microstructure responsible for the scattering. Unfortunately, the characteristic size measurement has traditionally been corrupted by uncertainties in the frequency-dependent attenuation along the propagation path. Recently, this problem was addressed by estimating the characteristic size and attenuation along the propagation path simultaneously using an algorithm termed the Spectral Fit (SF) algorithm. Using computer simulations to study the SF algorithm, the accuracy and precision of the attenuation estimate improved as the frequency range (largest frequency minus smallest frequency used by the algorithm when obtaining estimates) was increased. Similarly, the accuracy and precision of the scatterer size estimates were improved as the $\Delta k a_{\text {eff }}$ (largest wavenumber times scatterer radius minus smallest wavenumber times scatterer radius used by the algorithm) was increased. In this study, the simulation results were validated using a tissue mimicking phantom. The phantom had an attenuation of $0.83 \mathrm{~dB} / \mathrm{cm}-\mathrm{MHz}$ with glass beads with a radius range between 22.5 and $26.5 \mu \mathrm{m}$ at a concentration of $47.7 / \mathrm{mm}^{3}$. The SF algorithm accurately estimated the size of the glass beads and the precision of the size and attenuation estimates improved with increasing $\Delta k a_{\text {eff }}$ and frequency range respectively in the same manner as was observed in the simulation studies
\end{abstract}

Key words: Effective scatterer radius, Attenuation estimation, Frequency range 


\section{INTRODUCTION}

In the past, many different investigators have worked at extending the diagnostic capabilities of medical ultrasound by providing quantitative measures of tissue properties based on an analysis of the backscattered RF waveforms. One tissue property that has shown diagnostic potential is the effective radius, $a_{\text {eff }}$, of the tissue microstructure (Lizzi et al. 1983; Lizzi et al. 1986; Insana et al. 1990). Oelze et al. (2004) showed that the tissue microstructure could distinguish between fibroadenomas and carcinomas. Likewise, Tateishi et al. (1998) demonstrated that the tissue microstructure could be used to diagnose axillary lymph node metastases in breast cancer, and Feleppa et al. $(1996 ; 1997)$ used tissue microstructure to identify cancer in the prostate. As well as these examples involving tumors, Insana et al. (1995) used the properties of the tissue microstructure obtained from backscattered ultrasound signals to measure the structural properties of the kidney.

Despite these successes, determining $a_{\text {eff }}$ on a clinical basis has had only limited success due to the patient specific frequency-dependent attenuation along the propagation path, $\sim \alpha f$ where $f$ is frequency and $\alpha$ is the attenuation coefficient, masking the frequency dependence of $a_{\text {eff }}$ in the backscattered RF waveforms. The earlier successes were obtained when $\alpha$ could be measured or reasonably estimated a priori. Recently, we proposed a new algorithm, termed the Spectral Fit (SF) algorithm, that would overcome this shortcoming by estimating $a_{\text {eff }}$ and $\alpha$ simultaneously (Bigelow and O'Brien, submitted). Using computer simulations, we demonstrated that the accuracy and precision of the $a_{\text {eff }}$ estimate improved as the $\Delta k a_{\text {eff }}$ (largest wavenumber, $k$, minus the smallest wavenumber multiplied by $\left.a_{e f f}\right)$ used to obtain the estimate was increased. Similarly, the accuracy and precision of the $\alpha$ estimate improved as the frequency range (largest frequency minus the smallest frequency) used to obtain the estimate was increased.

In this work, we wanted to verify our simulation results by using the SF algorithm to analyze the backscattered RF waveforms from a glass bead tissue-mimicking phantom. In addition, we wanted to explore the impact the initial frequency/initial $k a_{\text {eff }}$ value used to obtain the estimates had on the accuracy and precision of the SF algorithm also using the waveforms from the glass bead phantom.

\section{REVIEW OF SF ALGORTIHM}

Before proceeding with the discussion, the SF algorithm will be reviewed. Assuming weakly focused sources and small window lengths, the 
expected backscattered voltage spectrum returned from a tissue region containing weak scatterers satisfying the Born approximation is given by (Bigelow and O'Brien, submitted)

$$
E\left[\left|V_{\text {scat }}(f)\right|^{2}\right] \propto k^{4}\left|V_{\text {plane }}(f)\right|^{2} e^{-4 \alpha f_{T}} F_{\gamma}\left(k a_{\text {eff }}\right)
$$

where $V_{\text {plane }}(f)$ is the voltage spectrum returned from a reference plane placed at the focal plane, $z_{T}$ is the distance from the region of interest (ROI) to the aperture plane of the transducer, and $F_{\gamma}\left(k a_{\text {eff }}\right)$ is the form factor that depends on the scatterer geometry. The geometry of the scatterer must be known before an accurate estimate of $a_{\text {eff }}$ can be obtained.

The SF algorithm solves for $a_{\text {eff }}$ and $\alpha$, by finding the values that minimize

$$
A S D=\operatorname{mean}_{f}\left[\left(X\left(f, a_{e f f}, \alpha\right)-\bar{X}\left(a_{e f f}, \alpha\right)\right)^{2}\right]
$$

where

$$
\begin{aligned}
& X\left(f, a_{\text {eff }}, \alpha\right)=\ln \left(E\left[\left|V_{\text {scat }}(f)\right|^{2}\right]\right)-\ln \left(k^{4}\left|V_{\text {plane }}(f)\right|^{2} e^{-4 \alpha f z_{T}} F_{\gamma}\left(k a_{\text {eff }}\right)\right) \\
& \bar{X}\left(a_{\text {eff }}, \alpha\right)=\operatorname{mean}_{f}\left[X\left(f, a_{\text {eff }}, \alpha\right)\right] .
\end{aligned}
$$

In the algorithm, an $E\left[\left|V_{\text {scat }}(f)\right|^{2}\right]$ is obtained by

$$
E\left[\left|V_{\text {scat }}(f)\right|^{2}\right] \cong \frac{1}{N} \sum_{i=1}^{N}\left(\left|V_{i}(f)\right|^{2}\right)
$$

where $N$ was 25 in our initial evaluation of the algorithm. Subtracting by the modified $\left|V_{\text {plane }}(f)\right|^{2}$ term in $X$ removes the system dependence of the backscattered power spectra. Also, subtracting by $\bar{X}$ removes the effects of any multiplicative constants allowing estimation of $a_{\text {eff }}$ and $\alpha$ independent of the acoustic concentration (Insana et al., 1990). 


\section{SIMULATION AND EXPERIMENTAL SETUP}

The simulations (Bigelow and O'Brien, submitted) used a spherically focused source with an f-number of 4 and a focal length of $5 \mathrm{~cm}$. The voltage spectrum returned from a plane placed at the focal plane for the simulated source was given by

$$
\left|V_{\text {plane }}(f)\right| \propto|f|^{2} \exp \left(-2\left(\frac{f-f_{o}}{\sigma_{o}}\right)^{2}\right)
$$

where $f_{o}$ was $8 \mathrm{MHz}$ and $\sigma_{o}$ was varied as 2,4 , and $6 \mathrm{MHz}$ to change the bandwidth of the source. The source exposed an infinite homogeneous halfspace where the attenuation of the half-space was varied from 0 to $1 \mathrm{~dB} / \mathrm{cm}$ $\mathrm{MHz}$ to test the impact of attenuation. The half-space contained Gaussian scatterers, $F_{\gamma}\left(k a_{e f f}\right)=\exp \left(-0.827\left(k a_{\text {eff }}\right)^{2}\right)$, of the same size at a density of $35 / \mathrm{mm}^{3}$ where $a_{e f f}$ varied from 5 to $150 \mu \mathrm{m}$ to test the impact of scatterer size. For each attenuation, scatterer size, and source bandwidth, the simulations generated and analyzed 1000 waveforms whose power spectrum were averaged in sets of 25 when determining $E\left[\left|V_{\text {scat }}(f)\right|^{2}\right]$ for a total of 40 estimates of $a_{\text {eff }}$ and $\alpha$ per case. Also, prior to the analysis, the waveforms were time gated with a Hamming window corresponding to $3 \mathrm{~mm}$ about the focus.

The phantom experiment also used a spherically focused source with an fnumber of 4 and a focal length of $5.35 \mathrm{~cm}$. The focus of the transducer was positioned $2 \mathrm{~cm}$ into the phantom. The transducer used in the experiment had a center frequency of $9.4 \mathrm{MHz}$ and a $-3 \mathrm{~dB}$ bandwidth of $3.8 \mathrm{MHz}$ as measured from the reflection off of a wire placed at the focus, and a $f_{o}$ value of $6.9 \mathrm{MHz}$ and a $\sigma_{o}$ value of $9.0 \mathrm{MHz}$ as described by Eqn. (5) when measured from the reflection off of a plane placed at the focal plane. The phantom had an attenuation of $0.83 \mathrm{~dB} / \mathrm{cm}-\mathrm{MHz}$ and contained glass beads, $F_{\gamma}\left(k a_{\text {eff }}\right) \cong\left[\sin \left(2 k a_{\text {eff }}\right) /\left(2 k a_{\text {eff }}\right)\right]^{2}$, with radii between $22.5-26.5 \mu \mathrm{m}$ at a density of $47.7 / \mathrm{mm}^{3} .350$ waveforms were acquired from the phantom and time gated with a Hamming window corresponding to $3 \mathrm{~mm}$ about the focus. In the phantom experiment, the initial and final $k a_{\text {eff }}$ values used by the SF algorithm were manually selected. Once again, the waveforms were averaged in sets of 25 to obtain 14 estimates of for each set of $k a_{\text {eff }}$ values used by the SF algorithm. 


\section{RESULTS}

\subsection{Results for Initial $k a_{e f f}$ and Initial Frequency}

The first analysis performed on the phantom data was to vary the initial $k a_{\text {eff }}$ value used by the SF algorithm while maintaining a constant $\Delta k a_{\text {eff }}$ of 0.5 . The results for this analysis are shown in Figure 1(a)-1(d). The error in the $a_{e f f}$ estimate for the phantom experiment is based on a size of $24.5 \mu \mathrm{m}$, the median size for the glass beads.

(a)

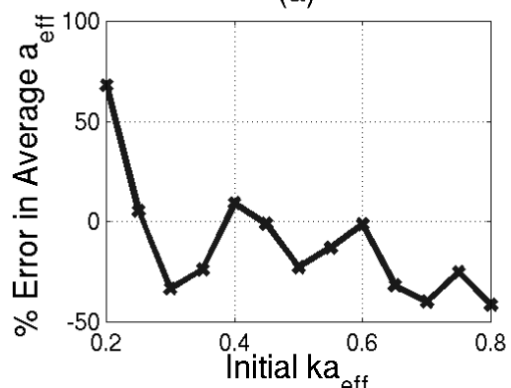

(c)

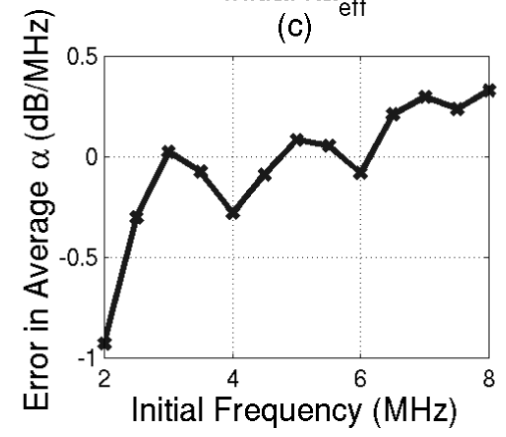

(b)

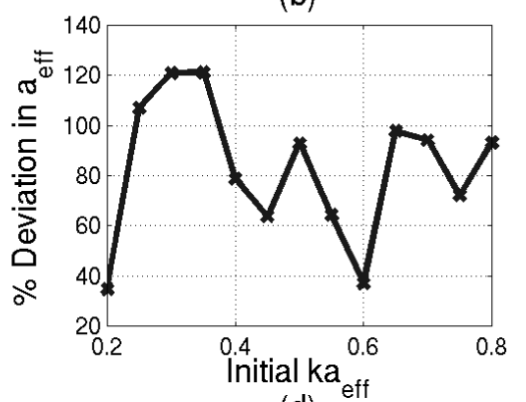

(d)

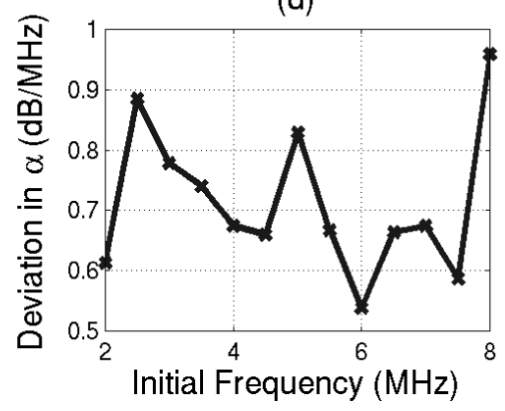

Figure 1. Phantom estimation results for different initial $k a_{\text {eff }}$ for a $\Delta k a_{\text {eff }}=0.5$. (a) Accuracy of $a_{\text {eff }}$ estimate, (b) precision of $a_{\text {eff }}$ estimate, (c) accuracy of $\alpha$ estimate, and (d) precision of $\alpha$ estimate.

The accuracy of the $a_{\text {eff }}$ and $\alpha$ estimates improves significantly as the initial $k a_{\text {eff }}$ is increased from 0.2 to 0.3 . After an initial $k a_{\text {eff }}$ of 0.3 , the accuracy of the estimates exhibits only a weak dependence on initial $k a_{e f f}$. The improvement at low initial $k a_{e f f}$ is probably due to the weak frequency dependence of $F_{\gamma}\left(k a_{e f f}\right)$ at low values of $k a_{e f f}$. 


\subsection{Results for $\Delta k a_{e f f}$ and Frequency Range}

After exploring the importance of the initial $k a_{\text {eff }}$ value used by the SF algorithm, the significant impact of $\Delta k a_{\text {eff }}$ and frequency range as observed in the earlier simulation study was verified using the phantom waveforms. This time the initial $k a_{e f f}$ was maintained at 0.3 while $\Delta k a_{e f f}$ was varied from 0.2 to 1 . The results from the phantom experiment are shown with a summary of the results from the earlier simulation study in Figure 2(a)-2(d).

(a)

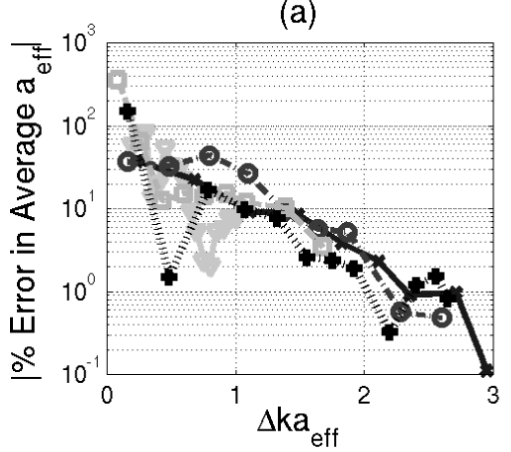

(c)

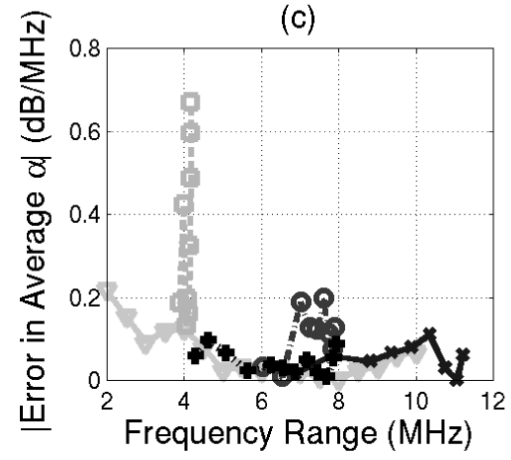

(b)

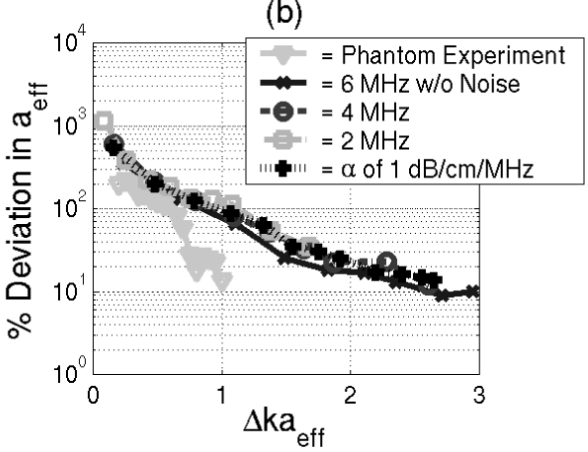

(d)

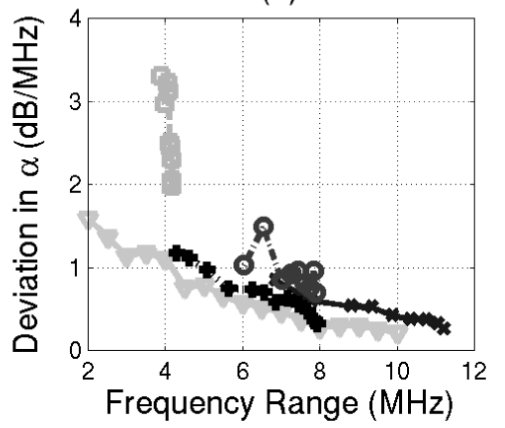

Figure 2. Results for different $\Delta k a_{\text {eff }}$ and frequency ranges from Phantom experiment $\left({ }^{\nabla}\right)$ and simulations, $\sigma_{o}$ of $6 \mathrm{MHz}$ and $\alpha$ of $0 \mathrm{~dB} / \mathrm{cm}-\mathrm{MHz}(*), \sigma_{o}$ of $4 \mathrm{MHz}$ and $\alpha$ of $0 \mathrm{~dB} / \mathrm{cm}$ $\mathrm{MHz}\left(-\sigma^{-}\right), \sigma_{o}$ of $2 \mathrm{MHz}$ and $\alpha$ of $0 \mathrm{~dB} / \mathrm{cm}-\mathrm{MHz}(-), \sigma_{o}$ of $6 \mathrm{MHz}$ and $\alpha$ of $1 \mathrm{~dB} / \mathrm{cm}-$ $\mathrm{MHz}$ (-4-). (a) Accuracy of $a_{\text {eff }}$ estimate, (b) precision of $a_{e f f}$ estimate, (c) accuracy of $\alpha$ estimate, and (d) precision of $\alpha$ estimate.

In both the simulation study and the phantom experiment there is improvement in the accuracy (Figure 2(a)) and precision (Figure 2(b)) of the $a_{\text {eff }}$ estimate with increasing $\Delta k a_{e f f}$, and improvement in the precision of the $\alpha$ estimate (Figure 2(d)) with increasing frequency range. However, the phantom 
results tend to be slightly more precise than the simulation results for the same $\Delta k a_{\text {eff }}$ and frequency range.

\section{CONCLUSIONS}

The SF algorithm can estimate both $a_{\text {eff }}$ and $\alpha$ simultaneously as was demonstrated by phantom experiments and computer simulations. Also, the accuracy of the SF algorithm exhibits only weak dependence on the initial $k a_{e f f}$ value for initial $k a_{e f f}>0.3$ as was illustrated by the phantom experiment. However, the accuracy and precision of the of the $a_{\text {eff }}$ estimate drastically improves with increasing $\Delta k a$ eff while the precision of the attenuation estimate drastically improves with increasing frequency range. The estimates obtained with the glass bead phantom exhibit the same trends observed in the Gaussian scatterer simulations but with improved precision. The improved precision is probably due to the stronger $k a$ eff dependence of the form factor for the glass beads as compared to the form factor for Gaussian scatterers as is illustrated by the kaeff dependence for both form factors shown in Figure 3.

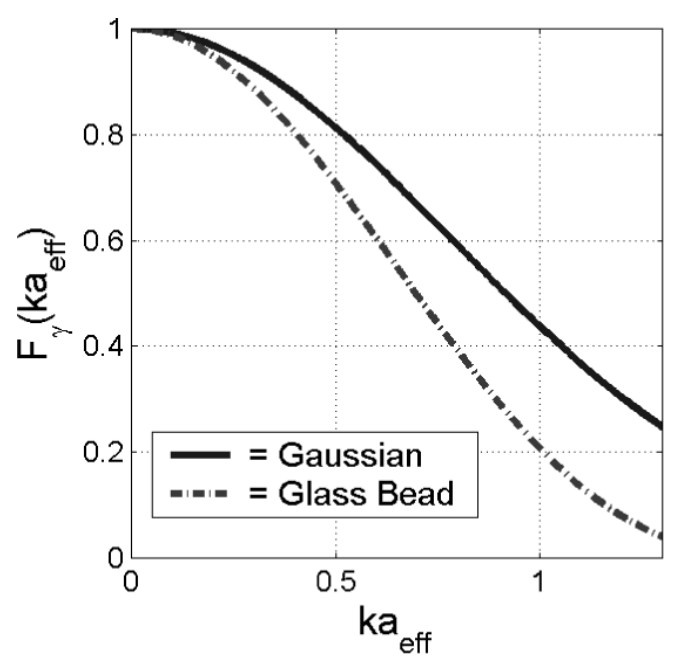

Figure 3. ka eff dependence of the form factor for the glass bead and Gaussian scatterer. 


\section{REFERENCES}

1. Bigelow, T.A., and O'Brien, W.D. Jr., submitted, Evaluation of the Spectral Fit algorithm as functions of frequency range and $\Delta \mathrm{ka}_{\mathrm{eff}}$, IEEE Trans. Ultrason. Ferroelect. Freq. Contr.

2. Feleppa, E.J., Kalisz, A., Sokil-Melgar, J.B., Lizzi, F.L., Liu, T., Rosado, A., Shao, M.C., Fair, W.R., Wang, Y., Cookson, M.S., Reuter, V.E., and Heston, W.D.W., 1996, Typing of prostate tissue by ultrasonic spectrum analysis, IEEE Trans. Ultrason. Ferroelect. Freq. Contr. 43: 609-619.

3. Feleppa, E.J., Liu, T., Kalisz, A., Shao, M.C., Fleshner, N., Reuter, V., and Fair, W.R., 1997, Ultrasounic spectral-parameter imaging of the prostate, Int. J. Imaging Syst. Technol., 8: 11-25.

4. Insana, M.F., Wagner, R.F., Brown, D.G., and Hall, T.J., 1990, Describing small-scale structure in random media using pulse-echo ultrasound, J. Acoust. Soc. Am. 87: 179-192.

5. Insana, M.F., Wood, J.G., Hall, T.J., Cox, G.G., and Harrison, L.A., 1995, Effects of endothelin-1 on renal microvasculature measured using quantitative ultrasound, Ultrasound in Med. \& Biol. 21: 1143-1151.

6. Lizzi, F.L., Greenebaum, M., Feleppa, E.J., and Elbaum, M., 1983, Theoretical framework for spectrum analysis in ultrasonic tissue characterization, J. Acoust. Soc. Am. 73: 1366-1373.

7. Lizzi, F.L., Ostromogilsky, M., Feleppa, E.J., Rorke, M.C., and Yaremko, M.M., 1986, Relationship of ultrasonic spectral parameters to features of tissue microstructure, IEEE Trans. Ultrason. Ferroelectr. Freq. Control. 33: 319-329.

8. Oelze, M.L., O'Brien, W.D. Jr., Blue, J.P., and Zachary, J.F., 2004, Differentiation and characterization of rat mammary fibroadenomas and 4T1 mouse carcinomas using quantitative ultrasound imaging, IEEE Trans. Med. Imaging. 23: 764-771.

9. Tateishi, T., Machi, J., Feleppa, E.J., Oishi, R., Jucha, J., Yanagihara, E., McCarthy, L.J., Noritomi, T., and Shirouzu, K., 1998, In vitro diagnosis of axillary lymph node metastases in breast cancer by spectrum analysis of radio frequency echo signals, Ultrasound in Med. \& Biol. 24: 1151-1159. 